\title{
The Fast Collection of Radio Fingerprint for WiFi- Based Indoor Positioning System
}

\author{
Hung-Huan Liu \\ Dept. of Electronic Engin. \\ Chung Yuan Christian University \\ Chung Li, Taiwan, R.O.C. \\ hhliu@cycu.edu.tw
}

\author{
Chung-Wei Liao \\ Master Program in Commun. Engin. \\ Chung Yuan Christian University \\ Chung Li, Taiwan, R.O.C.
}

\author{
Wei-Hsiang Lo \\ Dept. of Electronic Engin. \\ Chung Yuan Christian University \\ Chung Li, Taiwan, R.O.C.
}

\begin{abstract}
Mobile communications and Internet have become one of the most important services nowadays. Most mobile devices, such as cell phone, PDA, and laptop computer, equipped with the WiFi, GPS, and Bluetooth as their standard, build-in equipment, and people have got used to use mobile services in their daily life. One interesting service is Wifi-based indoor positioning system (IPS) that attracts many researchers devote their effort to it. Many researches in IPS determine the user location by the method of scene analysis. This method needs to collect the RSSI of APs from the interested place beforehand to build the building's WiFi radio fingerprint database and this task is time-consuming. Meanwhile, it also needs to resample very often in order to keep the accuracy of the positioning result. In this paper, we design and test a fast setup algorithm for collecting those sampling information. The Android smartphone and its build-in motion sensors were used to help collecting the AP's RSSI. We use the motion sensor to detect the pace while walking and collect the AP's RSSI in every step. The method gets through the sampling work in a walking duration which is much shorter than traditional method. This paper also compares the fast setup method with the traditional method from the positioning accuracy point of view. Experiments show that there is no significant difference on positioning accuracy between them.
\end{abstract}

Keywords-WiFi; Indoor Positioing System, IPS; Smartphone; motion sensor; radio-map

\section{INTRODUCTION}

During the last decade, the growth of wireless sensor network technologies have led to several new applications which allow location-based service (LBS) available to better serve its user according to user's context and location. Among these, WiFi-based indoor positioning and navigation services have attracted a lot of attention. It is because the system installation cost is lower than others in nowadays and offers acceptable positioning accuracy for some location-based services. WiFi, currently, is widely used in many territories in such a way that access point (AP) is very cheap and easy to obtain. WiFi AP has been widely deployed in many building, campus, and underground facilities. Meanwhile, the computation capacity of $\mathrm{WiFi}$ embedded smart mobile devices, such as iPhone and Android phone, gain ground rapidly and the cost is down continuously along with the fast development of personal mobile applications and services.

WiFi-based positioning methods are currently divided into three main positioning principles: proximity, trilateration, and scene analysis. Proximity positioning method regard the AP with strongest received signal strength indication (RSSI) as the user's location Such an algorithm is relatively simple; even though it has a fast positioning speed, its accuracy is lower when compared to other methods [1]. In general, positioning error is related to the density of AP provisioning. In addition, the APs' position should be known beforehand.

The trilateration positioning method uses three or more APs' location to calculate distance using time differences or signal strength in signal receptions, which are then used to estimate the location of the user. The current WiFi system can only work with the RSSI method, which uses a wireless transmission attenuation model to calculate the distance from the estimation point to the AP. The multi-path effect generated from wireless signals in an indoor environment has made it much more difficult for the trilateration method to achieve high-accuracy positioning, which results in failure to obtain acceptable levels of accuracy and precision. Some Researchers have dedicated their effort to improve the positioning accuracy based on trilateration[2][3].

The scene analysis positioning is generally divided into two phases. In the first phase, so-called offline phase, APs' RSSI and basic service set identification (BSSID) and the coordination of the sample point are recorded to build a radio fingerprint database in a building. In the second phase, called position estimation phase, user collects the AP's RSSI and BSSID, using smartphone, and compare them with the records in the fingerprint database in order to estimate the user's position. Nearest Neighbors in Signal Space (NNSS) algorithm is proposed in [4] for computing the distance of signal space between the observed and recorded measurements. The advantage of this approach is that it can reduce multi-path problems[5]. However, the intensity of sample points of this method directly affects positioning accuracy.

A crucial problem for the scene analysis positioning is the cost on the offline radio-fingerprint building, which is a timeconsuming task. In the past, when doing those data collection, we need to build up a coordination system for the building, using the tape measure to mark the position of every sample point and then perform the signal sampling at every simple point one by one.

In traditional method, a sampling process is going to scan the WiFi signal several times and then take the average as the final record for fingerprint in each sampling point. Sometimes, in

This work was supported by Ministry of Sci. and Tech. of Taiwan, R.O.C. under Grants MOST 103-2221-E-033 -045 and MOST 103-2632-E$033-001$-. 
order not to influent received signals because of human body, we need to sample the signal from the 4 different directions and then averaging them as the final record [4]. In a building, increasing the number of sampling points will directly increase the positioning accuracy of the scene analysis IPS method. However, it also increases the time used to build-up the fingerprint database.

We wonder if, when build the fingerprint database, the number of scan at each sampling point is reduced to be one, then the fingerprint database can suit for scene analysis or not? In this paper, we use the smartphone and its build-in motion sensor to implement a fast fingerprint database build-up algorithm for WiFi-based IPS. We are not the first one to use the motion sensor of smartphone on the WiFi-based IPS. The most famous one is the WiFiSlam, a startup company acquired by Apple in early 2013. Other researches, such as [6][7], have also been show the possibilities. However, our objective is to propose and verify a procedure to build the radio fingerprint in a short time from the implementation point of view. Meanwhile the motion sensor is only used in the phase of data collection.

The remaining sections of this paper are as follows: second section describes the system environment in today's wifi IPS and data collection method. Experiments are presented in the third section. Finally, conclusion is given in the last section.

\section{ENVIRONMENT DESCRIPTION AND FAST DATA COLLECTION}

Although the fingerprinting based floor estimation methods perform well, they suffer several problems in today's WiFi usage environment as follows: a) due to the free use of $\mathrm{WiFi}$, large amount of $\mathrm{WiFi}$ AP have been installed/uninstalled without administration; b) due to the use of smartphone bridge, there are some fake APs will be detected when performing offline fingerprint creation and online position determination; c) due to the wireless signal coverage and the convenience of network wiring problems, the location of AP's installation position concentrates at certain areas, such as the majority of AP locate at center of corridors but none locates at staircases. These problems force us to refine our method to fit the more complicated environment.

Through our observation, the number of APs in the Electrical Building in CYCU is 58 when we first scan this venue in mid2011. However, the number of APs increases sharply up to 289 APs in early-2014 and is up to an incredible number of 496 APs in mid-2014. The Electrical Building is an 8-floor building with about 60 meters long and 45 meters wide, obviously the distribution of APs is very crowded. At any place of the building and performing the AP scan, there are at least 40 APs can be found. Those include the APs used in the infrastructure of the campus wireless LAN, the APs used as the WiFi IP router in the rooms and labs in the building, and the APs used as specific devices such as print server. As we know, there are only 11 channels (in most place) for the $2.4 \mathrm{GHz} \mathrm{WiFi}$ and only 3 channels (channel 1, 6, and 11) can operate fully isolate, the more APs were installed, the less spectrum efficient were obtained. But with the facts of convenient and unrestricted use of $\mathrm{WiFi}$ and poor planning of campus WLAN, resulting in the grotesque disappointing field. Similar problem happened at $5 \mathrm{GHz}$ band. We think that the Electrical Building is not the only place having the crowed APs installation. It can be imaged that the situation happened in many campus and office blocks.

Because there are at most 3 isolated channels of WiFi in 2.4 $\mathrm{GHz}$ band, APs contend the channel for serving their user and mask effect happened when sampling the APs' beacon used for indoor position. Figure 1 shows the RSSI of APs on a sampling point located at 4 floor corridor of Electrical Building. In this sample, there are 180 scans, with one second gap, are collected. Note that there are only 8 APs was shown in this figure for simplicity and all of them are use the WiFi channel $1(2.412 \mathrm{GHz}$ band). It can be found that the signal of AP3 are not detected until the second minute because of the signal masking effect. It is also shown that the APs with weaker RSSI have more probable shaded by others.

The second problem is the use of Smartphone Bridge. More and more user uses Smartphone as a hotspot to bridge WiFi signal to $3 \mathrm{G} / 4 \mathrm{G}$ network for accessing the Internet. If someone is using the WiFi IPS but having some others use the Smartphone Bridge around she/him, it will impact on the accuracy of positioning. Problems a) and b) may be solved by refine the IPS algorithm, however, the problem c) can not.

Figure 2 shows the strongest RSSI distribution in 4 floor of Electrical Building. The strongest RSSI at every sample point is the one which be scanned and stronger than others. The sample points in this test includes staircases and corridors, from the left side to the right side of the building with one-meter gap. The range of the indexed horizontal axis is from -3 to 58 meter. The emitting power of APs, using for campus WLAN, is around $-27 \mathrm{dBm}$ (measured at one meter distance), and about $-32 \mathrm{dBm}$ for WiFi IP router, so the RSSI higher than $-45 \mathrm{dBm}$ implies that the sample point is very close to an AP. On the other hand, the RSSI below than $-55 \mathrm{dBm}$ implies there is no AP setup around the sample point. From Figure 2, although more than 100 APs can be detected in this floor, we can deduce that there is no AP installed in staircases. Moreover, it can be deduced that the signal in region 1 marked in Fig. 2 is dominated by APs which used for campus WLANs; in region 2, the signal maybe be dominated by APs which used for WiFi IP routers installed in labs; in region 3, there is no AP installed. The ununiformed deployment of AP results in trouble when performing RSSIbased position determination algorithm at the place where close to the staircase.

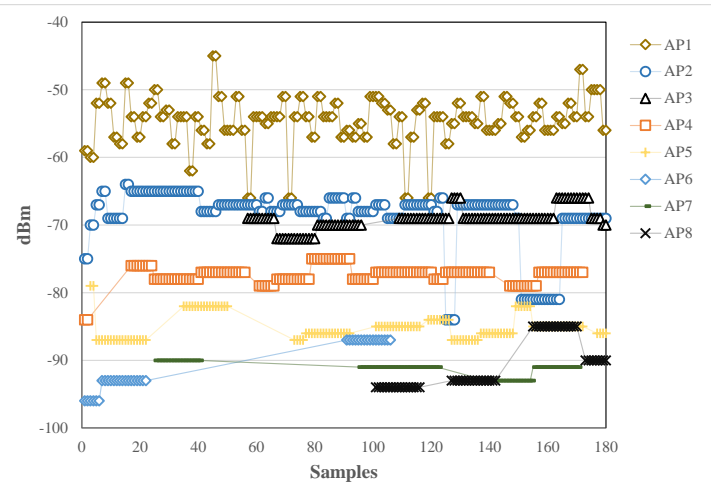

Fig. 1. RSSI sequence at a sample point 


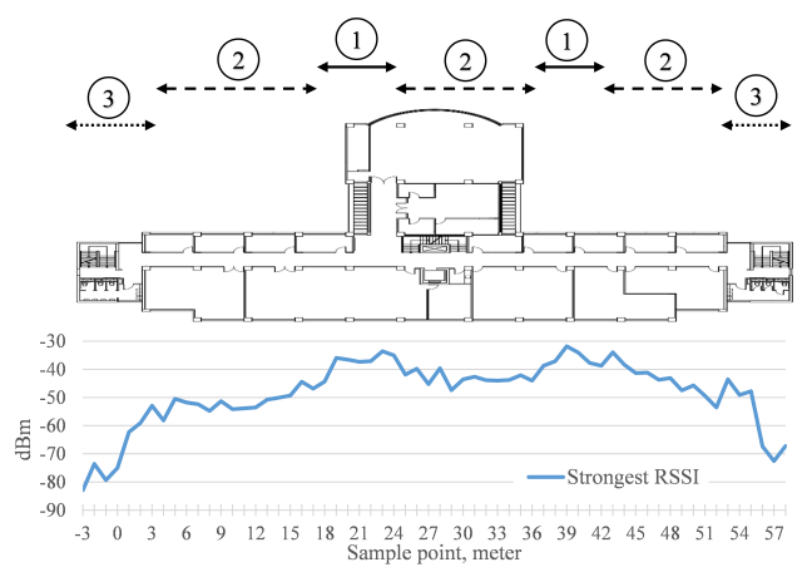

Fig. 2. The strongest RSSI distribution at 4 floor

The proposed system is divided into two phases: offline data collection phase and online position estimation phase. But the source of information and procedure involving to the offline data collection phase is illustrated in Fig. 3 (a), and the online position estimation phase's procedure is illustrated in Fig. 3 (b).

The simple idea of the offline data collection phase is that: A user who holds an Android smartphone walks from one side to the other side of the venue. In every step, the smartphone performs a scan and records the scanned RSSI and BSSID of surrounding APs. Note that, there is only one scan at each step, so there is probability that some AP's information will miss due to the mask effect discussed above. Sensor events and the map are used for producing the coordinate of the sampling point. From Android KitKat (Android 4.4) the SDK provides two new step detector and counter sensors but under supporting of specific hardware. As a consequence, we perform step detection based on the build-in low-cost microelectromechanical system (MEMS)-based inertial sensors in smartphone[8]. However, those methods cannot response the step event in time. For example, the step sensor in Android KitKat needs few seconds to report the event (The latency is expected to be below 2

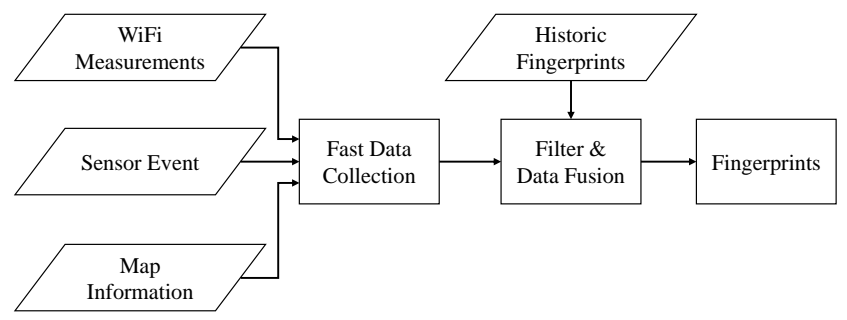

(a)

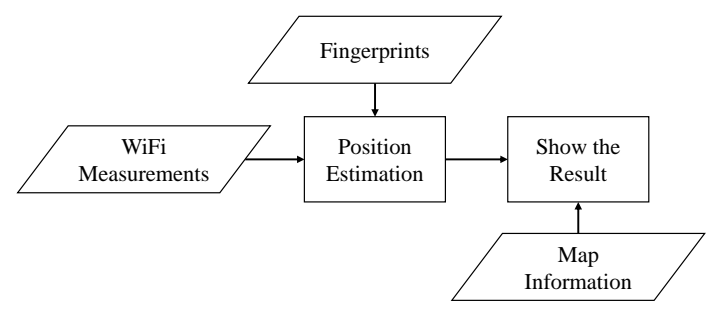

Fig. 3. (a) Offline fingerprint creation, (b) position estimation seconds stated in the official document). For the sake of response to the step detection accuracy and in time, we implement our own step detection algorithm on Android smartphone in constriction of limiting user's walking to be steady and stable in data collection phrase. The overall offline procedure is shown in Fig. 4.

\section{EXPERIMENT AND RESULT}

\section{A. Experiment Environment}

In order to verify the usability of the proposed method, two different data collection methods, called static sampling (SS) and moving sampling (MS), are preformed and compared. The testing place is at the 4th floor in the Electrical Building in CYCU. The sampling path are illustrated in Fig. 5 (a), two straight lines are sampled by SS and MS, respectively. The SS collects data by sampling the surrounding APs' RSSI and BSSID along the straight lines and at every one meter. In each sample, 30 scans are performed and then take the average RSSI to be the RSSI in fingerprint database. The APs which the number of RSSI to be detected less than 10 will be filtered out from the database. We use a tripod to mount the smartphone to do the sampling in order to ignore the effect of absorption of radio signal by human body. In the experiment, the HTC one X based on Android 4.1.2 OS smartphone is used. On the other hand, the MS collects data by the method discussed in Sec. 2.

The fingerprint database is a 2-tuple of AP RSSI measurements and the corresponding media access control (MAC) addresses:

$$
S_{j k}=\left\{\left(b_{i j k}, s s_{i j k}\right) \mid 1 \leq i\right\} .
$$

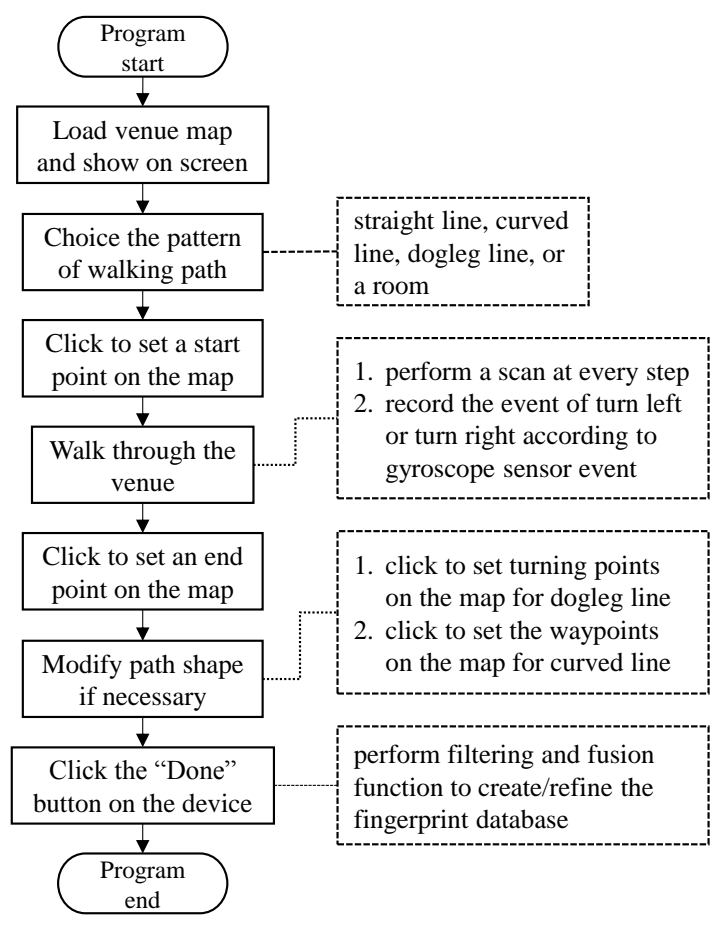

Fig. 4. Offline sampling procedure and its actions 


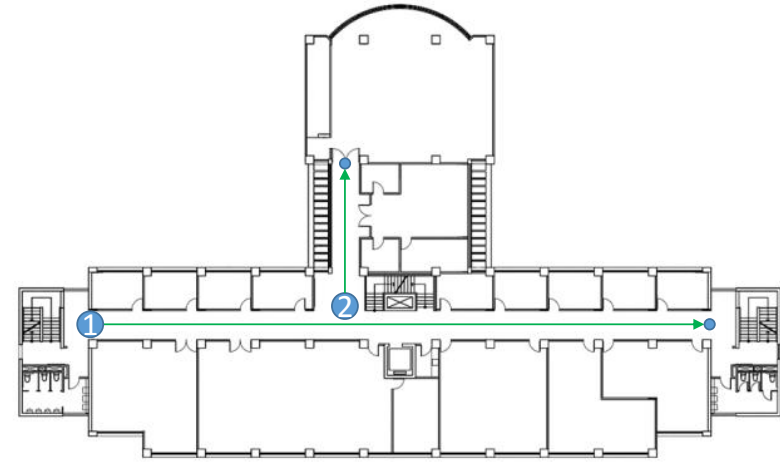

(a)

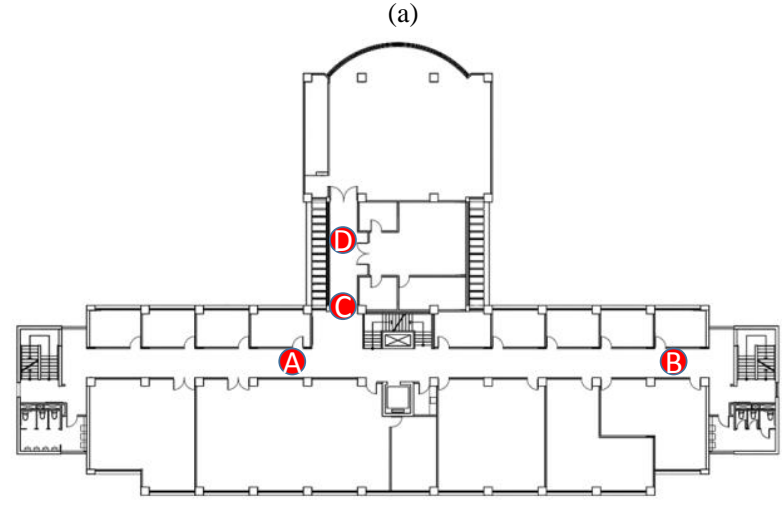

(b)

Fig. 5. (a) Two sampling paths for experiments, (b) four testing points

Suffix $k$ means the sample point is on $k$ th path and $j$ means it is the $j$ th sample on $k^{\text {th }}$ path. $b_{i j k}$ is the $i^{\text {th }}$ AP's BSSID and $s s_{i j k}$ is the $i^{\text {th }}$ RSSI of $j^{\text {th }}$ sample in order of RSSI on the $k^{\text {th }}$ path, respectively.

\section{B. Position estimation method}

Four points at the corridor are randomly chosen to be the testing point for comparing the MS and SS, which are shown in Fig. 5 (b) which coordinates are $(20,3),(49,3),(23,7)$, and (23, 13) from A to D, respectively. A Minimum Signal Distance with Relational Factor algorithm, called MSDRF, is used to estimate the position of the testing points and is as follows:

Let $u=\left\{\left(b_{i}, s s_{i}\right) \mid 1 \leq i \leq p\right\}$ be the measured APs' information of a testing point in descendant order of RSSI, where $p$ is a predefined threshold and is set to be 6 in this experiment, i.e., the most strongest 6 APs' information measured at the testing point were included in the set $u$. The distance between the testing point and a sampling point is defined as:

$$
\left\{\begin{array}{c}
\left|u-S_{j k}\right|, \quad u \text { and } S_{j k} \text { are related } \\
\infty, \quad u \text { and } S_{j k} \text { are unrelated }
\end{array}\right.
$$

The reason to put a relational factor as a constraint is that if the number of sample point increases, the number of unrelated sample point is also increasing, too many unrelated sample points will impact on the result of position estimation. Relational factor, denoted as $r$, is defended as the number of AP's BSSID both in $u$ and in $S_{j k}$ for determining if a sampling point is "relative" to the testing point or not. For example, let $r=2, p=$ 3 , and let $u=\left\{\left(b_{a}, s s_{u, a}\right)\left(b_{b}, s s_{u, b}\right)\left(b_{c}, s s_{u, c}\right)\right\}$, sampling point $S_{1}$ $=\left\{\left(b_{a}, s s_{s l, a}\right),\left(b_{b}, s s_{s l, b}\right),\left(b_{d}, s s_{s l, d}\right),\left(b_{e}, s s_{s l, e}\right)\right\}$, and sampling point $S_{2}=\left\{\left(b_{c}, s s_{s 2, c}\right),\left(b_{d}, s s_{s 2, d}\right),\left(b_{f}, s s_{s 2, f}\right)\right\}$. Then $S_{1}$ is "relative" to $u$ because $b_{a}$ and $b_{b}$, are both in $u$ and $S_{1}$, but $S_{2}$ is not "relative" to $u$, because there is only one BSSID $b_{c}$ is in $u$ and $S_{2}$, which the number is less than $r$. We also define the $r / p$ as the relational coefficient. It expresses a rough filter to filter the sampling points where far from the testing point without join into the calculation. Besides, it expresses the ability to adopt the phenomenon of AP's signal shading at a single scan in the MS model. The RSSI distance $\left|u-S_{1}\right|$ is defined as:

$$
\sqrt{\left(S S_{u, a}-S S_{s 1, a}\right)^{2}+\left(S S_{u, b}-S S_{s 1, \mathrm{~b}}\right)^{2}+\left(T_{\min }\right)^{2}}
$$

where $T_{\min }$ is a constant used to instead of the mismatch AP when calculating the signal distance between $u$ and $S_{1}$.

\section{Experiment Results}

The experiment results are shown in Fig. 6 to Fig. 9 for the 4 different testing points and for the SS and MS fingerprint database respectively. The vertical axis is the signal distance from the testing point to the sampling point evaluated from eq. (2). Because the fingerprint is created for the experiment, there is no filter and fusion are performed to refine the database. Consequently, some signal distances vary a lot in this experiment. The red spot on the horizontal axis presents the position of the testing point. There is a polynomial regression drawn on these figures. It can be found that the local minimum value of the SS and MS are close. That means we can use the MS in place of SS in the WiFi-based IPS.

\section{CONCLUSION}

In this paper, two offline fingerprint collection methods are performed and compared. Our goal is to find a way to speed up the creation and maintenance for the offline data collection phase for the wifi-based IPS. Experiments show that combining the build-in motion sensor of the smartphone and venue map the MS method can largely reduce the setup time of the IPS. In the meantime, the MS method perform almost close performance to the SS method. It can be thought that the MS method will be the main method for the signal sampling in the future IPS.

\section{REFERENCES}

[1] A. H. Sayed, A. Tarighat, and N. Khajehnouri, "Network-based wireless location: challenges faced in developing techniques for accurate wireless location information." IEEE Signal Processing Magazine, vol. 22, issue 4, July 2005, pp. 24-40.

[2] H. H. Liu, W. H. Lo, C. C. Tseng, and H. Y. Shin, "A WiFi-Based Weighted Screening Method for Indoor Positioning Systems," Wireless Pers. Commun. vol. 79, no. 1, 2014, pp. 611-627.

[3] Y. Gwon, and R. Jain. "Error characteristics and calibration-free techniques for wireless LAN-based location estimation." in Proc. of the second international workshop on Mobility management \& wireless access protocols, pp. 2-9. ACM, 2004.

[4] P. Bahl and V. N. Padmanabhan, "RADAR: an in-building RF-based user location and tracking system," in Proc. IEEE INFOCOM 2000, pp. 775784, May 2000.

[5] H. Liu, H. Darabi, P. Banerjee, and J. Liu, "Survey of Wireless Indoor Positioning Techniques and Systems," IEEE TSMCC, vol. 6, issue 6, Nov. 2007, pp. 1067-1080. 


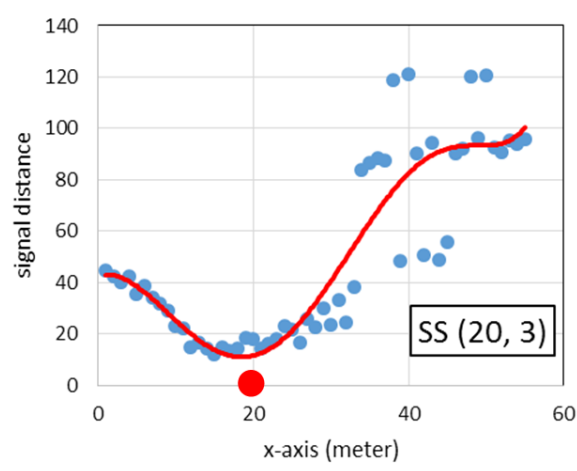

(a)

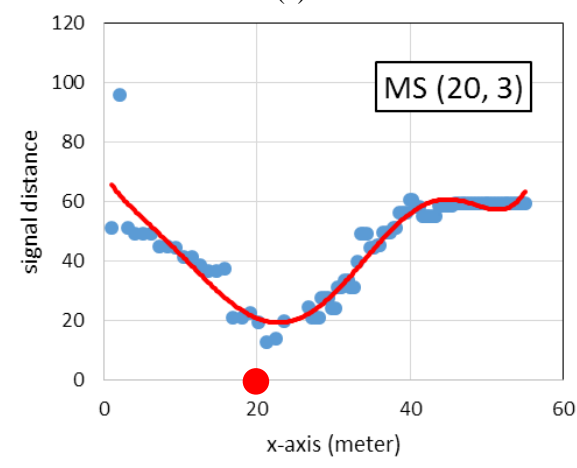

(b)

Fig. 6. The experiment result for the testing point (20, 3) (a) SS method, (b) MS method

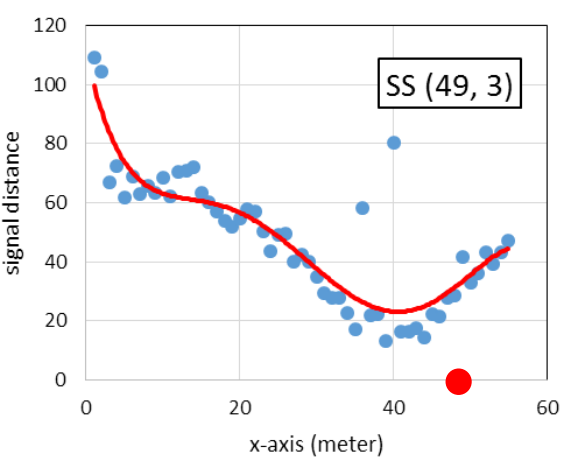

(a)

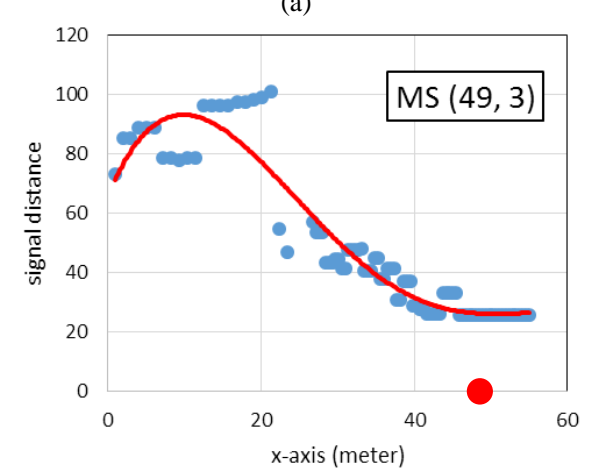

(b)

Fig. 7. The experiment result for the testing point $(49,3)$ (a) SS method, (b) MS method
[6] A. Naguib, P. Pakzad, R. Palanki, S. Poduri, and Y Chen, "Scalable and Accurate Indoor Positioning on Mobile Devices," in 2013 International Conference on Indoor Positioning and Indoor Navigation, MontbeliardBelfort, France, Oct. 28- 31, 2013

[7] I. Constandache, R. R. Choudhury, and I. Rhee. "Towards mobile phone localization without war-driving." In Proc. IEEE INFOCOM 2010, pp. 19, 2010.

[8] J. Qian, J. Ma, R. Ying, P. Liu, and L. Pei, "An Improved Indoor Localization Method Using Smartphone Inertial Sensors," in 2013 International Conference on Indoor Positioning and Indoor Navigation, Montbeliard-Belfort, France, Oct. 28- 31, 2013

[9] H. Ying, C. Silex, A. Schnitzer, S. Leonhardt, M.Schiek, “Automatic Step Detection in the Accelerometer Signal," 4th International Workshop on Wearable and Implantable Body Sensor Networks, Springer Berlin Heidelberg, 2007.

[10] M. Z. Naqvi, A. Kumar, A. Chauhan, and K. Sahni, "Step Counting Using Smartphone-Based Accelerometer," IJCSE, vol. 4, no. 5, May 2012, pp. 675-681.

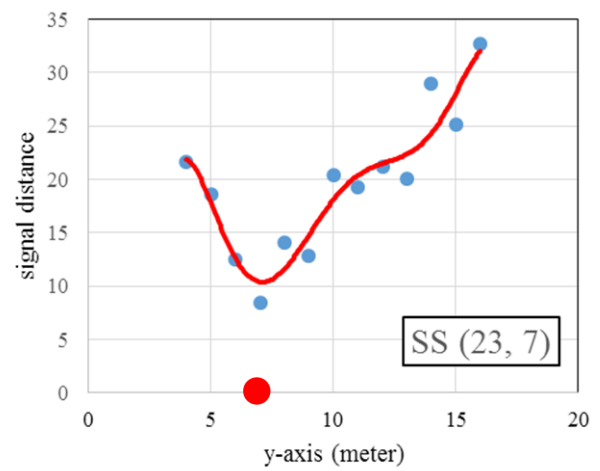

(a)

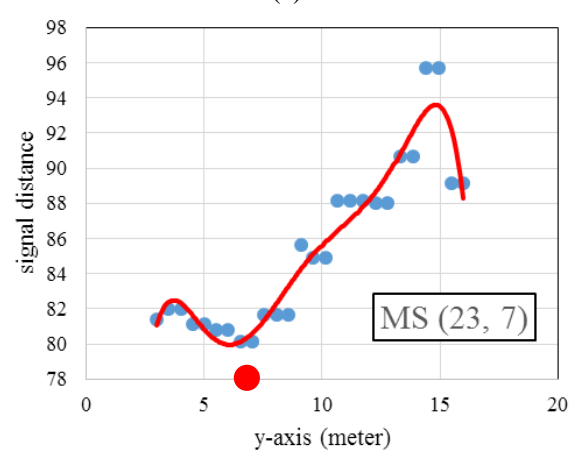

(b)

Fig. 8. The experiment result for the testing point $(23,7)$ (a) SS method, (b) MS method 


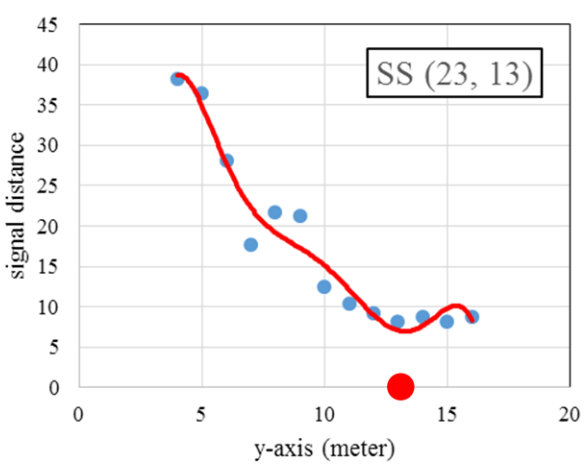

(a)

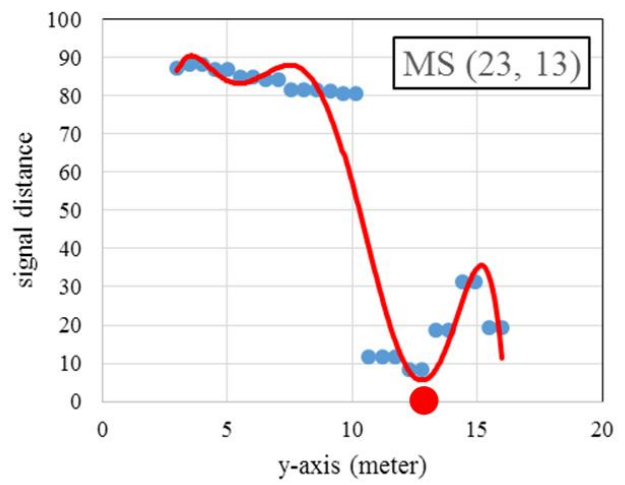

(b)

Fig. 9. The experiment result for the testing point $(23,13)$ (a) SS method, (b) MS method 